\title{
Medida da acuidade visual em um shopping center metropolitano
}

\section{Visual acuity measurement in a metropolitan shopping mall population}

Giovanni M. Travi (1)

Alexandre S. Marcon (1)

Marcelo N. Teixeira ${ }^{(1)}$

Terla N. de Castro ${ }^{(1)}$

Zélia M. Correa ${ }^{(2)}$

Italo M. Marcon ${ }^{(3)}$

\section{RESUMO}

Objetivo: Determinar a prevalência de baixa acuidade visual (AV) em uma amostra populacional, com um padrão socioeconômico diferenciado.

Métodos: Realizou-se estudo de prevalência, sendo verificada a AV de 213 voluntários examinados em um posto no interior de um shopping center em Porto Alegre, por meio do aparelho Ortho-Rater, da Bausch \& Lomb. Foi medida a AV para longe em todos os indivíduos e naqueles com 40 anos ou mais, a AV para perto, ambos sem e com correção. Determinou-se como baixa $A V$, aquela inferior a 10 (equivalente a 20/20 na Tabela de Snellen).

Resultados: A idade variou de 6 a 75 anos, com uma média \pm desviopadrão de 27,8 $\pm 15,16$ anos, pertencendo a maioria dos examinados à faixa entre 10 e 40 anos. Encontrou-se uma prevalência de 38,7\% (IC 95\%: 32,1-45,7) de baixa AV para longe nos dois olhos. 24,9\% (IC 95\%: 19,2-31,3) apresentavam déficit visual binocular e não faziam uso de correção. Para perto, observou-se que 70,8\% (IC 95\%: 55,983) dos indivíduos testados apresentaram déficit visual binocular sem correção e $41,7 \%$ (IC 95\%: 27,6-56,8) apresentaram baixa AV independente do uso de correção.

Conclusão: Este estudo demonstrou que existe considerável parcela da população com déficit visual, necessitando de avaliação e manejo adequados. Conclui-se que programas de medida da AV e orientação sobre saúde ocular são importantes em qualquer ambiente e atingindo diferentes classes socioeconômicas.

Palavras-chave: Acuidade visual; Triagem populacional; Promoção da saúde.

\section{INTRODUÇ̃̃O}

Congresso Brasileiro de Prevenção da Cegueira, Rio de Janeiro, RJ, em 7 de setembro de 1998.

(1) Médicos residentes do curso de especialização em oftalmologia da Irmandade da Santa Casa de Misericórdia de Porto Alegre (ISCMPA), Fundação Faculdade Federal de Ciências Médicas de Porto Alegre (FFFCMPA), Porto Alegre, RS, Brasil.

(2) Médica responsável pelo setor de Retina do Serviço de Oftalmologia da ISCMPA, FFFCMPA, Porto Alegre, RS, Brasil.

(3) Médico chefe do serviço e coordenador do curso de especialização em oftalmologia da ISCMPA, FFFCMPA. Professor regente da disciplina de oftalmologia da FFFCMPA, Porto Alegre, RS, Brasil.

Os autores declaram que não possuem interesse financeiro no desenvolvimento ou marketing dos instrumentos referidos neste estudo.

Endereço para correspondência: Giovanni M. Travi. Rua Felicíssimo de Azevedo, 264, apto. 603. Porto Alegre (RS). CEP 90540-110. e-mail: gmtravi@ conex.com.br

\begin{tabular}{l}
\hline INTRODUÇ̃̃o \\
\hline A acuidade visual (AV) é determinada pela menor imagem retiniana \\
percebida pelo indivíduo. Sua medida é dada pela relação entre o tamanho \\
do menor objeto (optotipo) visualizado e a distância entre observador e \\
objeto ${ }^{1}$. A diminuição da acuidade visual causa importante déficit funcio- \\
nal e considerável morbidade a seus portadores. Seu reconhecimento é \\
importante, pois na maior parte das vezes tal deficiência pode ser corrigida \\
com terapêutica adequada ${ }^{2,3}$. A AV de uma forma geral, está relacionada \\
com a idade e o nível socioeconômico da população ${ }^{2,4}$. Trabalhos demons- \\
tram níveis inferiores de AV em idosos, bem como em populações com \\
situação socioeconômica menos favorecida ${ }^{5-8}$. \\
O objetivo deste trabalho foi determinar a prevalência de baixa AV em \\
amostra populacional com padrão socioeconômico diferenciado. Para tan-
\end{tabular}


to, foi medida a AV em um posto instalado num shopping center na zona norte de Porto Alegre. O local fica distante do centro da cidade, e é freqüentado por pessoas de todas as faixas etárias, preferencialmente pelas classes econômicas média à alta (classes A e B).

\section{PACIENTES \& MÉTODOS}

Este estudo foi realizado durante um projeto de orientação e conscientização da população em relação aos seus cuidados com a saúde, promovido pela Irmandade da Santa Casa de Misericórdia de Porto Alegre.

Foi verificada a AV em um posto montado num shopping center em Porto Alegre no período de 19 de junho a 3 de julho de 1997. A população havia sido alertada a respeito da campanha através da mídia, sendo a participação espontânea.

A acuidade visual foi medida com o aparelho Ortho-Rater, da Bausch \& Lomb. Este método gradua a AV em uma escala crescente de 0 a 12, apresentando o optotipo 10 equivalência ao 20/20 da tabela de Snellen. A melhor acuidade visual obtida em cada olho foi registrada. Considerou-se como AV normal valores iguais ou superiores à 10 , estabelecendo-se como déficit de $\mathrm{AV}$ valores inferiores a este.

O exame iniciava-se com um questionário, com dados de identificação, idade, sexo, realização de consulta oftalmológica prévia, patologias oculares passadas e uso de correção óptica. Em seguida, verificava-se a AV do olho direito (OD) e após do olho esquerdo (OE). A visão era testada para longe e, em indivíduos com 40 anos ou mais, para perto, respeitando a ordem descrita. Pessoas usuárias de óculos, eram testados também com correção. Usuários de lentes de contato eram testados apenas com correção.

Foram excluídos do estudo menores de 6 anos de idade pela dificuldade técnica na realização do exame.

Os examinadores foram médicos residentes e estagiários do Serviço de Oftalmologia da ISCMPA, previamente treinados.

Todos os indivíduos avaliados foram orientados a consultar um oftalmologista.

Os dados foram descritos através de média e desviopadrão para variáveis quantitativas e percentuais para os dados qualitativos. Os intervalos de confiança para os percentuais apresentados foram baseados na distribuição binomial. A análise foi realizada com o auxílio do programa Epi-Info, versão 6.03 .

\section{RESULTADOS}

Foram avaliadas 213 pessoas voluntárias em um shopping center, sendo $55,1 \%$ (114) do sexo feminino. A média \pm desvio-padrão da idade foi de 27,8 $\pm 15,16$, com uma amplitude de 6 a 75 anos, pertencendo a maioria dos examinados entre 10 e 40 anos.

Das 213 pessoas examinadas, 60,1\% (128) já haviam con- sultado um oftalmologista previamente e 23,9\% (51) informavam fazer uso de correção (óculos ou lentes de contato). Todos os pacientes utilizando correção informavam consulta oftalmológica prévia.

Quando indagados sobre patologias prévias, $23,9 \%$ (51) referiram já ter tido alguma alteração ocular. As ametropias perfizeram a quase totalidade das alterações referidas $(88,7 \%)$, sendo o restante determinado por patologias palpebrais não especificadas $(3,8 \%)$, retinopatia $(3,8 \%)$, catarata $(1,9 \%)$ e glaucoma $(1,9 \%)$.

A acuidade visual para longe sem correção foi verificada em 209 pacientes, e com correção em 20 pacientes. A média de acuidade visual sem correção foi de 8,4 $\pm 2,8$ para OD e 8,6 2 2,7 para OE. A acuidade visual dos pacientes usuários de correção teve média de 7,1 $\pm 3,6$ para o OD e de $6,9 \pm 3,6$ para o OE.

A análise da AV sem correção demonstrou que $48,3 \%$ (IC 95\%: 41,4-55,3) apresentaram AV menor que $10 \mathrm{em}$ OD e 45,9\% (IC 95\%: 39-52,9) em OE. 38,7\% (IC 95\%: 32,1-45,7) da amostra apresentaram AV menor que 10 nos dois olhos (AO), sem correção. Destes 81 pacientes, $35,8 \%$ (29) informaram ser usuários de óculos, sendo que 13 portavam os óculos na ocasião do exame e apenas 5 deles apresentavam AV de 10 em pelo menos um dos olhos, quando testados com correção. 3 pacientes usuários de lentes de contato foram testados só para longe com correção, sendo que apenas um deles tinha AV de 10 em pelo menos um dos olhos.

Verificou-se que 24,9\% (IC 95\%: 19,2-31,3) apresentaram $\mathrm{AV}$ abaixo de 10 nos dois olhos e não faziam uso de correção, e que 4,8\% (IC 95\%: 2,3-8,6) apresentaram AV abaixo do normal nos dois olhos, mesmo usando correção. Dos sujeitos examinados, 29,7\% (IC 95\%: 23,6-36,3) mostraram-se com AV abaixo do normal nos dois olhos, independente do uso de correção. Este cálculo admite que os usuários de óculos que não os portavam no momento do exame, estariam com AV normal quando em uso dos mesmos. Caso estes indivíduos apresentem AV abaixo do normal, mesmo com correção, haveria uma porcentagem superior de baixa AV para longe (Tabela 1).

\begin{tabular}{|c|c|}
\hline \multicolumn{2}{|c|}{ no de voluntários } \\
\hline AV p/ longe sem correção & $(n=213)$ \\
\hline Baixa AV OD & $101(48,3)$ \\
\hline Baixa AV OE & $96(45,9)$ \\
\hline Baixa AV AO & $81(38,7)$ \\
\hline $\begin{array}{l}\text { Melhor AV p/ longe, independente do } \\
\text { uso de correção }\end{array}$ & $(n=212)$ \\
\hline AV normal em AO sem correção & $128(60,4)$ \\
\hline AV normal em AO com correção & $6(2,8)$ \\
\hline Baixa AV AO, não usuários de óculos & $52(24,9)$ \\
\hline Baixa AV AO, usuários de óculos, com correção & $10(4,8)$ \\
\hline $\begin{array}{l}\text { Baixa AV AO, usuários de óculos, } \\
\text { não testados com correção }\end{array}$ & $16(7,5)$ \\
\hline
\end{tabular}


Analisando-se a distribuição da AV para longe sem correção conforme faixa etária, observou-se um declínio da média da AV com o avançar da idade. Segundo nossos achados, estima-se que a perda de acuidade visual no grupo estudado seja de 0,5 pontos (IC 95\%: 0,3-0,7; $<<0,05$ ) para cada 10 anos (Gráfico 1).

A AV para perto foi avaliada em 48 pacientes com 40 anos ou mais. Demonstrou-se que a AV sem correção para perto para OD teve média de 5,6 $\pm 3,5$ e OE foi de 5,6 $\pm 3,7$. Com correção observou-se para OD média de $8,0 \pm 3,0$ e para $\mathrm{OE}$ de $7,5 \pm 3,1$.

Das 48 pessoas testadas para perto sem correção, $75 \%$ (IC 95\%: 60,4-85,9) apresentavam AV menor que $10 \mathrm{em}$ OD e 77,1\% (IC 95\%: 62,7-88) em OE. 70,8\% (IC 95\%: 55,9-83) da amostra apresentaram AV para perto, sem correção menor que 10 em ambos os olhos. Destes 34, 64,7\% (22) referiram uso de óculos para perto, sendo que apenas 14 portavam seus óculos na ocasião do exame, e foram testados com correção. Destes 14, 7 pacientes estavam com $\mathrm{AV}$ menor que 10 para perto nos dois olhos com correção, e 7 apresentavam AV igual ou maior que 10 pelo menos em um dos olhos (Tabela 2). Observou-se que 43,7\% (IC 95\%: 29,5-58,8) apresentavam AV normal em pelo menos um dos olhos, não levando-se em consideração o uso de correção. Já 41,7\% (IC 95\%: 27,6-56,8) estavam com deficiência visual para perto, sendo que até mesmo os que utilizavam correção para perto apresentaram esta deficiência. 14,6\% (7) não foram testados para perto com correção pois não portavam a mesma no momento do exame.

\section{DISCUSSÃO}

Este estudo revelou uma prevalência de baixa acuidade visual binocular para longe em torno de $40 \%$. Observou-se que aproximadamente $25 \%$ dos indivíduos apresentaram baixa AV para longe nos dois olhos e não faziam uso de correção. Além disto, 4,7\% apresentaram baixa visual mesmo em uso de correção. Estes dados sugerem desinformação e talvez relativa difi-

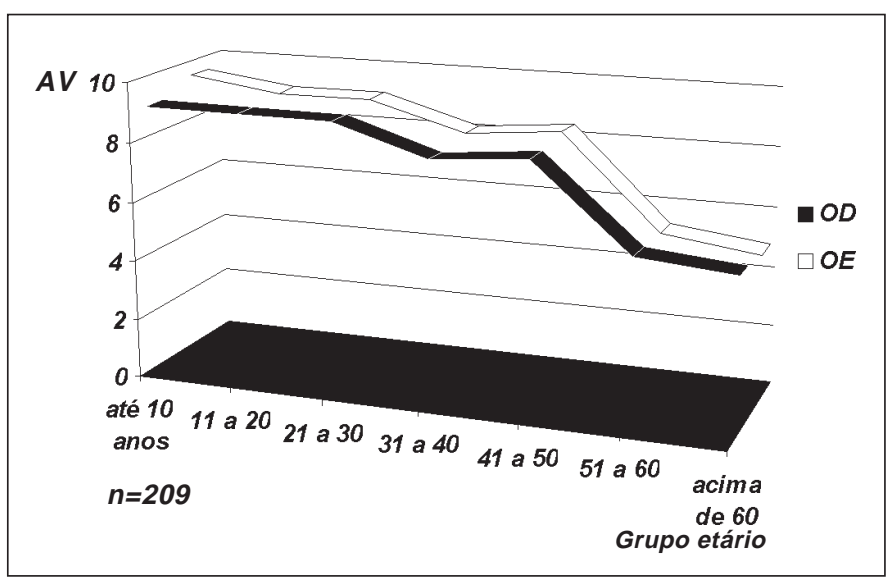

Gráfico 1 - Distribuição da média da acuidade visual para longe, sem correção, conforme a faixa etária.

\begin{tabular}{|c|c|}
\hline & no de voluntários \\
\hline AV p/ perto sem correção & $(n=48)$ \\
\hline Baixa AV OD & $36(75)$ \\
\hline Baixa AV OE & $37(77,1)$ \\
\hline Baixa AV AO & $34(70,8)$ \\
\hline AV p/ perto com correção & $(n=14)$ \\
\hline Baixa AV OD & $8(57,1)$ \\
\hline Baixa AV OE & $7(50)$ \\
\hline Baixa AV AO & $7(50)$ \\
\hline $\begin{array}{l}\text { AV p/ perto independente do } \\
\text { uso de correção }\end{array}$ & $(n=48)$ \\
\hline $\begin{array}{l}\text { AV normal AO com ou sem } \\
\text { correção em uso }\end{array}$ & $21(43,7)$ \\
\hline $\begin{array}{l}\text { Baixa AV AO com ou sem } \\
\text { correção em uso }\end{array}$ & $20(41,7)$ \\
\hline $\begin{array}{l}\text { Baixa AV AO, usuários de óculos, } \\
\text { não testados com correção }\end{array}$ & $7(14,6)$ \\
\hline
\end{tabular}

culdade de acesso aos cuidados oftalmológicos. Considerando que $66 \%$ da população estudada estava na faixa etária dos 10 aos 40 anos, e que na população jovem, as ametropias lideram como causa de baixa de visão, podemos inferir que a maior parte desta baixa visual poderia ser corrigida. Estudo semelhante realizado em um departamento de trânsito, mostrou que $79,2 \%$ dos candidatos reprovados no exame de acuidade visual, eram portadores de ametropias não corrigidas ${ }^{2}$.

Com o passar da idade, a prevalência das doenças oculares aumenta e o espectro das doenças que atingem o olho muda. A ametropia deixa de ser a causa mais importante de baixa de visão, aumentando a incidência de catarata, doença macular senil, glaucoma e outras ${ }^{4,9}$. Neste estudo, verificou-se que o envelhecimento da população contribui para a diminuição da AV média para longe.

Analisando-se a AV para perto nas pessoas com mais de 40 anos, verificou-se uma grande incidência $(70,8 \%)$ de déficit visual para perto, em função das alterações acomodativas comuns nesta faixa etária ${ }^{1}$. Apesar desse déficit, uma parcela significativa $(27,1 \%)$ não usa óculos para perto e $41,7 \%$ dos indivíduos com mais de 40 anos (contando os que usam e que não usam óculos) mesmo em uso de óculos apresentaram-se com baixa AV para perto. Isto pode ser decorrência da desinformação, baixa necessidade visual ou dificuldades econômicas.

Outro achado importante foi a boa acuidade visual para longe presente nesta população $(63,2 \%)$ (com e sem correção). $\mathrm{O}$ fato deste estudo ter sido realizado em local freqüentado principalmente por pessoas das classes A e B pode explicar esse achado, uma vez que estudos revelam que a prevalência de baixa acuidade visual aumenta em populações de baixa renda ${ }^{5,6}$. Em estudo publicado, foi encontrado uma prevalência de cegueira de 1,2\% na Inglaterra. Usando os mesmos critérios para a cegueira, esse índice subiu para 7,7\% 
no Quênia, revelando a relação entre baixa acuidade visual, qualidade de vida e, por conseqüência, poder aquisitivo da população ${ }^{5}$.

O presente estudo mostrou que existe uma considerável parcela da população com redução da acuidade visual potencialmente corrigível. Considerando-se o bom nível socioeconômico dos freqüentadores do local onde foi realizado este estudo, poder-se-ia especular que nas classes menos favorecidas economicanente, este índice de baixa AV seria ainda maior. Entretanto deve-se considerar que muitos voluntários provavelmente se submeteram ao teste por já apresentarem algum déficit visual. Desta forma estes valores tenderiam a estar superestimados.

Conclui-se que programas de medida da acuidade visual e orientação sobre saúde ocular são importantes veículos de informação em qualquer ambiente e atingindo as diferentes classes socioeconômicas.

\section{SUMMARY}

Purpose: To determine the prevalence of low visual acuity (VA) in a populational sample, with a differentiated social pattern. Methods: This was a prevalence study, in which the 213 volunteers' VA, examined in a shopping mall in Porto Alegre, was verified through the Ortho-Rater device, from Bausch \& Lomb. Far sight VA was measured in all the individuals and in those who were 40 years old or older, near sight VA, both of them without and with correction. VA was considered low when it was less than 10 (equivalent to 20/20 on the Snellen chart).

Results: The age group varied from 6 to 75 years, presentig a mean \pm standard deviation of $27.8 \pm 15.16$ years, most belonging to the range between 10 and 40 years. A prevalence of 38.7\% (IC 95\%: 32.1-45.7) of low far sight VA in both eyes was detected. $24.9 \%$ (IC 95\%: 19.2-31.3) presented binocular visual deficit and they didn't use any optic correction. For near sight VA, it was observed that 70.8\% (IC 95\%: 55.9-83) of the tested individuals presented binocular visual deficit without correction and 41.7\% (IC 95\%: 27.6-56.8) presented low VA, independent of the correction used.

Conclusion: This study demonstrated that a considerable part of the population has low visual acuity, needing adequate evaluation and handling. It is concluded that visual screening programs and ocular health orientation are important for any group and should reach different socioeconomic levels.

Keywords: Visual acuity; Mass screening; Health promotion.

\section{REFERÊNCIAS BIBLIOGRÁFICAS}

1. Duke-Elder S. Prática de refração em oftalmologia, $9^{\mathrm{a}}$ ed. Rio de Janeiro: Livraria Atheneu, 1984.

2. Ramos AR, Zaniolo S, Matsumoto LH, Ramos CCB, Ferreira RLS, Santos L, Viana JE, Moreira Jr. CA. Causas de reprovação no teste de acuidade visual do departamento de trânsito do Paraná. Arq Bras Oftalmol 1994;57(5):305-9.

3. Kamali A, Whitworth JA, Ruberantwari A, Mulwanyi F, Acakara M, Dolin P, Johnson G. Causes and prevalence of non-vision impairing ocular conditions among a rural adult population in sw Uganda. Ophthalmic Epidemiol 1999; 6(1):41-8.

4. Brandão EO, Oliveira NM, Pereira MVC, Maia NBF, Pereira GC. Acuidade visual de motoristas de veículos pesados numa rodovia de grande circulação Problemática e sugestões. Arq Bras Oftalmol 1995;58(2):121-6.

5. Foster A. Patterns of blindness, In: Duane, TD. Clinical Ophthalmology, Philadelphia, Harper \& How, 1984;5(53).

6. Medina NH, Barros OM, Ramos LR. Morbidade ocular em idosos da cidade de São Paulo - SP. Arq Bras Oftalmol 1993;56(5):276,81-83.

7. Ho JH, Chang RJ, Wheeler NC, Lee DA. Ophthalmic disorders among the homeless and nonhomeless in Los Angeles. J Am Optom Assoc 1997;68(9):567-73.

8. Van Newkirk MR. The Hong Kong vision study: a pilot assessment of visual impairment in adults. Trans Am Ophthalmol Soc 1997;95:715-49.

9. Rahmani B, Tielsch JM, Katz J, Gottsch J, Quigley H, Javitt J, Sommer A. The cause-specific prevalence of visual impairment in na urban population. Ophthalmology 1996;103(11):1721-6.

\section{Novidades na Internet!!!}

Agora no site CBO você tem disponível todas as informações na íntegra dos

Arquivos Brasileiros de Oftalmologia

http: / / www. cbo.com.br/abo 